Hrytsak Myroslava V., Popovych Dariya V., Badiuk Nataliya S., Hrytsan Ivanna I., Żukow Xawery. Comparative study of the effect on the neuroendocrine-immune complex and metabolism of drinking monotherapy with Naftussya water and therapy supplemented with "Myroslava" and "Khrystyna" mineral waters. Journal of Education, Health and Sport. 2022;12(1):355-367. eISSN 2391-8306. DOI http://dx.doi.org/10.12775/JEHS.2022.12.01.031

https://apcz.umk.pl/JEHS/article/view/JEHS.2022.12.01.031

https://zenodo.org/record/6026356

The journal has had 40 points in Ministry of Eduction and Science of Poland parametric evaluation. Annex to the announcement of the Minister of Education and Science of December 21, 2021. No 32343. Has a Journal's Unique Identifier: 201159. Scientific disciplines assigned: Physical Culture Sciences (Field of Medical sciences and health sciences); Health Sciences (Field of Medical Sciences and Health Sciences).

Punkty Ministerialne z 2019 - aktualny rok 40 punktów. Zalącznik do komunikatu Ministra Edukacji i Nauki z dnia 21 grudnia 2021 r. Lp. 32343. Posiada Unikatowy Identyfikator Czasopisma: 201159. Przypisane dyscypliny naukowe:Nauki o kulturze fizycznej (Dziedzina nauk medycznych i nauk o zdrowiu); Nauki o zdrowiu (Dziedzina nauk medycznych i nauk o zdrowiu).

(1) The Authors 2022;

This article is published with open access at Licensee Open Journal Systems of Nicolaus Copernicus University in Torun, Poland

Open Access. This article is distributed under the terms of the Creative Commons Attribution Noncommercial License which permits any noncommercial use, distribution, and reproduction in any medium, provided the original author (s) and source are credited. This is an open access article licensed under the terms of the Creative Commons Attribution Non commercial license Share alike. The authors declare that there is no conflict of interests regarding the publication of this paper.

Received: 06.01.2022. Revised: 06.01.2022. Accepted: 31.01.2022.

\title{
Comparative study of the effect on the neuroendocrine-immune complex and metabolism of drinking monotherapy with Naftussya water and therapy supplemented with "Myroslava" and "Khrystyna" mineral waters
}

\author{
Myroslava V. Hrytsak ${ }^{1,2}$, Dariya V. Popovych ${ }^{3}$, Nataliya S. Badiuk ${ }^{1,4}$, \\ Ivanna I. Hrytsan ${ }^{1,4}$, Xawery Żukow ${ }^{5}$
}

${ }^{1}$ SE Ukrainian Research Institute for Medicine of Transport, Odesa, Ukraine badiuk_ns@ukr.net

${ }^{2}$ Scientific group of Balneology of Hotel\&Spa Complex "Karpaty", Truskavets', Ukraine hrytsak.myroslava@gmail.com ira barschyk@ukr.net

${ }^{3}$ IY Horbachevs'kyi National Medical University, Ternopil', Ukraine darakoz@yahoo.com ${ }^{4}$ International Medical University, Odesa, Ukraine

${ }^{5}$ Medical University of Bialystok, Bialystok, Poland xaweryzukow@gmail.com

Background. Earlier in an experiment on rats, we showed that the newly created sulfatechloride sodium-magnesium drinking mineral waters of Truskavets' spa have neuroendocrine and metabolic effects significantly different from daily water. Adhering to the principle "From experiment to clinic", we continued research in this direction with the participation of patients of the resort. Materials and Methods. The object of clinical-physiological observation were 34 men aged 23-70 years, who underwent rehabilitation treatment of chronic cholecystitis and pyelonephritis in remission in the Truskavets resort. The examination was performed twice, before and after a 7-10-day course of balneotherapy. All patients received bioactive water Naftussya, however, 11 men additionally drank water "Khrystyna", and the other 11 men - water "Myroslava". The subject of the study were the parameters of the neuroendocrine-immune complex and metabolism. Results. The complex balneotherapy by interval use of sulfate-chloride sodium-magnesium mineral waters with Naftusya water causes significant changes in the constellation of neuroendocrine, metabolic and immune parameters, which are different from the effects of Naftusya water monotherapy. Own effects of mineral waters are estimated by modeling. In general, the effects are physiologically favorable and have a normalizing nature. Conclusion. The newly created sulfate-chloride sodium-magnesium drinking mineral waters of Truskavets resort have favorable neuroendocrine, metabolic and immune effects on patients with chronic cholecystitis and pyelonephritis. 
Key words: sulfate-chloride sodium-magnesium drinking mineral waters, Truskavets' spa, neuroendocrine, metabolic and immune parameters.

\section{INRODUCTION}

Earlier in an experiment on rats, we showed that the newly created sulfate-chloride sodium-magnesium drinking mineral waters of Truskavets' spa have neuroendocrine and metabolic effects significantly different from daily water [11-13]. Adhering to the principle "Ex experimento ad clinic", we continued research in this direction with the participation of patients of the resort.

\section{MATERIALS AND METHODS}

The object of clinical-physiological observation were 34 men aged 23-70 years, who underwent rehabilitation treatment in the Truskavets resort of chronic cholecystitis and pyelonephritis in remission with of neuroendocrine-immune complex dysfunction. The examination was performed twice, before and after a 7-10-day course of balneotherapy. All patients received bioactive water Naftussya $(3 \mathrm{ml} / \mathrm{kg}$ one hour before meals three times a day), however, 11 men in half an hour additionally drank water "Khrystyna", and the other 11 men - water "Myroslava" in the same dose.

The day before, daily urine was collected, in which was determined the concentration of electrolytes: calcium (by reaction with arsenase III), magnesium (by reaction with colgamite), phosphates (phosphate-molybdate method), chloride (mercury-rhodanidine method), sodium and potassium (flamming photometry); nitric metabolites: creatinine (by Jaffe's color reaction by Popper's method), urea (urease method by reaction with phenolhypochlorite), uric acid (uricase method). Urine lithogenicity index (Lith) was also calculated by the Tiselius' HS [24] formula modifed by Flyunt VR et al [7]:

Lith $=(\text { Uric acid } \bullet \text { Calcium/Magnesium } \bullet \text { Creatinine })^{0,25}$.

The same metabolic parameters were determined in plasma as well as glucose (glucoseoxidase method), triglycerides (by a certain meta-periodate method), total cholesterol (by a direct method after the classic reaction by Zlatkis-Zack) and content of him in composition of $\alpha$-lipoproteins (by the enzyme method after precipitation of not $\alpha$-lipoproteins); prae- $\beta$ lipoproteins (expected by the level of triglycerides); $\beta$-lipoproteins (expected by a difference between a total cholesterol and cholesterol in composition $\alpha$-and prae- $\beta$-lipoproteins).

The analysis carried out according to instructions [8] with the use of analyzers "Reflotron" (BRD) and "Pointe-180" (USA) and corresponding sets of reagents.

According to the parameters of $\mathrm{Ca}$ and phosphates exchange, parathyroid activity was evaluated by coefficient $(\mathrm{Cap} \cdot \mathrm{Pu} / \mathrm{Cau} \cdot \mathrm{Pp})^{0,25}$, based on its classical effects and recommendations by Popovych IL [9] as well as evaluated sympatho-vagal balance by coefficient $(\mathrm{Cap} / \mathrm{Kp})^{0,5}[6]$.

We determined content in plasma major hormones of adaptation: Cortisol, Testosterone and Triiodothyronine (by the ELISA with the use of analyzer "RT-2100C" and corresponding sets of reagents from “Алкор Био”, XЕМА Co., Ltd and DRG International Inc.).

In basal conditions we estimated the state of the autonomous regulation by the method heart rate variability (HRV) [1,3,10,23], using a hardware-programmatic complex "CardioLab+HRV" (KhAI Medica, Kharkiv, Ukraine). The following parameters were subject to analysis. Frequency Domain Methods: HF $(0,4 \div 0,15 \mathrm{~Hz}), \mathrm{LF}(0,15 \div 0,04 \mathrm{~Hz})$, VLF 
$(0,04 \div 0,015 \mathrm{~Hz})$, ULF $(0,015 \div 0,003 \mathrm{~Hz})$ компоненти. Time Domain Methods: HR, SDNN, RMSSD, $\mathrm{pNN}_{50}$. Calculated as well as Kerdö's Vegetative Index [5] and the entropy (h) of the relative spectral powers (SP) of the HRV bands by the formula Popovych IL [9]:

$\mathrm{hHRV}=-\left[\mathrm{SPHF} \bullet \log _{2} \mathrm{SPHF}+\mathrm{SPLF} \bullet \log _{2} \mathrm{SPLF}+\mathrm{SPVLF} \bullet \log _{2} \mathrm{SPVLF}+\mathrm{SPULF} \bullet \log _{2} \mathrm{SPULF}\right] / \log _{2} 4$

Immune status evaluated on a set of I and II levels recommended by the WHO as described in the manual [19]. For phenotyping subpopulations of lymphocytes used the methods of rosette formation with sheep erythrocytes on which adsorbed monoclonal antibodies against receptors CD3, CD4, CD8, CD22 and CD56 from company "Granum" (Kharkiv) with visualization under light microscope with immersion system. Subpopulation of $T$ cells with receptors high affinity determined by test of "active" rosette formation. The state of humoral immunity judged by the concentration in serum of Immunoglobulins classes G, A, M (ELISA, analyser "Immunochem", USA) and circulating immune complexes (by polyethylene glycol precipitation method) as well as C-reactive protein (by the ELISA with the use of analyzer "RT-2100C"), Interleukins $1 \beta$ and 6 (ELISA, analyzer "Stat Fax 303", USA, reagents from "Vector-Best", RF).

In portion of capillary the blood we counted up Leukocytogram and calculated the Entropy (h) of Leukocytogram (LCG) as well as Immunocytogram (ICG) using IL Popovych's formulas [9]:

$\mathrm{hLCG}=-\left[\mathrm{L} \bullet \log _{2} \mathrm{~L}+\mathrm{M} \bullet \log _{2} \mathrm{M}+\mathrm{E} \bullet \log _{2} \mathrm{E}+\mathrm{SNN} \bullet \log _{2} \mathrm{SNN}+\mathrm{StubN} \bullet \log _{2} \mathrm{StubN}\right] / \log _{2} 5$

$\mathrm{hICG}=-\left[\mathrm{CD} 4 \bullet \log _{2} \mathrm{CD} 4+\mathrm{CD} 8 \bullet \log _{2} \mathrm{CD} 8+\mathrm{CD} 22 \bullet \log _{2} \mathrm{CD} 22+\mathrm{CD} 56 \bullet \log _{2} \mathrm{CD} 56\right] / \log _{2} 4$

Parameters of phagocytic function of neutrophils estimated as described by SD Douglas and PG Quie [4] with moderately modification by MM Kovbasnyuk [18]. The objects of phagocytosis served daily cultures of Staphylococcus aureus (ATCC N 25423 F49) as typical specimen for Gram-positive Bacteria and Escherichia coli (O55 K59) as typical representative of Gram-negative Bacteria. Both cultures obtained from Laboratory of Hydro-Geological Regime-Operational Station JSC “Truskavets'kurort". Take into account the following parameters of Phagocytosis: activity (percentage of neutrophils, in which found microbes Hamburger's Phagocytic Index PhI), intensity (number of microbes absorbed one phagocytes - Microbial Count MC or Right's Index) and completeness (percentage of dead microbes Killing Index KI). On the basis of the recorded partial parameters of Phagocytosis, taking into account the Neutrophils $(\mathrm{N})$ content of 1 L blood, we calculated the integral parameter Bactericidal Capacity of Neutrophils $(\mathrm{BCCN})$ by the formula [9]:

$\mathrm{BCCN}\left(10^{9} \mathrm{Bact} / \mathrm{L}\right)=\mathrm{N}\left(10^{9} / \mathrm{L}\right) \bullet \mathrm{PhI}(\%) \bullet \mathrm{MC}(\mathrm{Bact} / \mathrm{Phag}) \cdot \mathrm{KI}(\%) \bullet 10^{-4}$

On the tone and motility of gall-bladder judged by its volume on an empty stomach in the morning and after 5, 15 and $30 \mathrm{~min}$ after ingestion cholekinetic $(50 \mathrm{ml}$ of $40 \%$ solution of xylitol). The method echoscopy (echocamera "Radmir") applicated [20,21]. To quantify cholekinetics, the area between the cholecystovolumogram and the basal line was calculated.

Normal (reference) values of variables are taken from the database of the Truskavetsian School of Balneology.

For statistical analysis used the software package "Statistica 64".

\section{RESULTS AND DISCUSSION}

Following the accepted algorithm, in this study, two research groups were combined to determine the effects common to both sulfate-magnesium mineral waters.

In order to identify those indicators for which the condition of patients on admission to treatment and after monotherapy or complex balneotherapy differ significantly, a discriminant analysis [16] of registered indicators was conducted. The program included in the discriminant model 27 variables, including 15 metabolic, 7 neuroendocrine and 5 immune (Tables 1 and 3). 
Table 1. Summary of the analysis of discriminant functions in relation to the parameters of metabolism and neuro-endocrine-immune complex

Step 27, N of vars in model: 27; Grouping: 3 grps; Wilks' $\Lambda$ : 0,022; approx. $F_{(57)}=7,9 ; p<10^{-6}$

\begin{tabular}{|c|c|c|c|c|c|c|c|c|c|}
\hline \multirow[b]{2}{*}{$\begin{array}{l}\text { Variables } \\
\text { currently in the } \\
\text { model }\end{array}$} & \multicolumn{3}{|c|}{ Groups (n) and Means \pm SE } & \multicolumn{5}{|c|}{ Parameters of Wilks' Statistics } & \multirow[b]{2}{*}{$\begin{array}{l}\text { Norm } \\
\text { Cv } \\
(30)\end{array}$} \\
\hline & $\begin{array}{l}\text { Before } \\
\text { thera- } \\
\text { py (34) }\end{array}$ & $\begin{array}{l}\text { After } \\
\text { Naftus- } \\
\text { sya (12) }\end{array}$ & $\begin{array}{l}\text { After Salt } \\
\text { Waters } \\
\text { and N (22) }\end{array}$ & $\begin{array}{l}\text { Wil } \\
\mathrm{ks} \\
\Lambda\end{array}$ & $\begin{array}{l}\text { Par- } \\
\text { tial } \\
\Lambda\end{array}$ & $\begin{array}{l}\text { F-re- } \\
\text { move } \\
(2,4)\end{array}$ & $\begin{array}{l}\text { p- } \\
\text { level }\end{array}$ & $\begin{array}{l}\text { Tole- } \\
\text { rancy }\end{array}$ & \\
\hline $\begin{array}{l}\text { Phosphates Exc- } \\
\text { retion, } \mathrm{mM} / 24 \mathrm{~h}\end{array}$ & $\begin{array}{l}18,2 \\
1,2\end{array}$ & $\begin{array}{l}16,8 \\
1,8 \\
\end{array}$ & $\begin{array}{l}42,4 \\
3,8 \\
\end{array}$ & 0,050 & 0,428 & 25,3 & $10^{-6}$ & 0,233 & $\begin{array}{l}25,2 \\
0,294 \\
\end{array}$ \\
\hline $\begin{array}{l}\text { Calcitonin, } \\
\mathrm{ng} / \mathrm{L}\end{array}$ & $\begin{array}{l}6,95 \\
0,62\end{array}$ & $\begin{array}{l}6,16 \\
1,11\end{array}$ & $\begin{array}{l}10,48 \\
1,21\end{array}$ & 0,029 & 0,747 & 6,42 & 0,004 & 0,427 & $\begin{array}{l}13,95 \\
0,493\end{array}$ \\
\hline $\begin{array}{l}\text { Creatinine Plasma, } \\
\mu \mathrm{M} / \mathrm{L}\end{array}$ & $\begin{array}{l}92,6 \\
2,6\end{array}$ & $\begin{array}{l}81,9 \\
2,8\end{array}$ & $\begin{array}{l}87,4 \\
2,0\end{array}$ & 0,033 & 0,654 & 10,1 & $10^{-3}$ & 0,426 & $\begin{array}{l}79,5 \\
0,167\end{array}$ \\
\hline $\begin{array}{l}\text { Testosterone, } \\
\mathrm{nM} / \mathrm{L}\end{array}$ & $\begin{array}{l}18,5 \\
1,6\end{array}$ & $\begin{array}{l}9,0 \\
1,0\end{array}$ & $\begin{array}{l}15,3 \\
2,1\end{array}$ & 0,036 & 0,602 & 12,6 & $10^{-4}$ & 0,359 & $\begin{array}{l}14,8 \\
0,400\end{array}$ \\
\hline $\begin{array}{l}\text { Sodium Plasma, } \\
\mathrm{mM} / \mathrm{L}\end{array}$ & $\begin{array}{l}141,5 \\
1,5\end{array}$ & $\begin{array}{l}146,7 \\
2,1\end{array}$ & $\begin{array}{l}142,3 \\
2,0\end{array}$ & 0,031 & 0,699 & 8,19 & 0,001 & 0,260 & $\begin{array}{l}145,0 \\
0,034\end{array}$ \\
\hline $\begin{array}{l}\text { Phosphate Plasma, } \\
\mathrm{mM} / \mathrm{L}\end{array}$ & $\begin{array}{l}1,04 \\
0,03\end{array}$ & $\begin{array}{l}1,13 \\
0,06\end{array}$ & $\begin{array}{l}0,91 \\
0,04\end{array}$ & 0,028 & 0,785 & 5,20 & 0,010 & 0,274 & $\begin{array}{l}1,20 \\
0,167\end{array}$ \\
\hline $\begin{array}{l}\text { Magnesium Urine, } \\
\mathrm{mM} / \mathrm{L}\end{array}$ & $\begin{array}{l}2,40 \\
0,11\end{array}$ & $\begin{array}{l}2,14 \\
0,23\end{array}$ & $\begin{array}{l}2,22 \\
0,13\end{array}$ & 0,027 & 0,816 & 4,28 & 0,021 & 0,095 & $\begin{array}{l}2,93 \\
0,256\end{array}$ \\
\hline $\begin{array}{l}\text { Chloride Excre-tion, } \\
\mathrm{mM} / 24 \mathrm{~h}\end{array}$ & $\begin{array}{l}186 \\
13\end{array}$ & $\begin{array}{l}197 \\
15\end{array}$ & $\begin{array}{l}259 \\
27\end{array}$ & 0,023 & 0,936 & 1,30 & 0,284 & 0,025 & $\begin{array}{l}167,5 \\
0,172\end{array}$ \\
\hline $\begin{array}{l}\text { Interleukin-6, } \\
\text { ng/L }\end{array}$ & $\begin{array}{l}4,45 \\
0,36 \\
\end{array}$ & $\begin{array}{l}3,67 \\
0,56 \\
\end{array}$ & $\begin{array}{l}4,58 \\
0,33 \\
\end{array}$ & 0,026 & 0,843 & 3,54 & 0,039 & 0,240 & $\begin{array}{l}4,25 \\
0,324 \\
\end{array}$ \\
\hline $\begin{array}{l}\text { LD Cholesterol } \\
\text { Plasma, } \mathrm{mM} / \mathrm{L}\end{array}$ & $\begin{array}{l}3,54 \\
0,18\end{array}$ & $\begin{array}{l}3,43 \\
0,32\end{array}$ & $\begin{array}{l}3,25 \\
0,21\end{array}$ & 0,032 & 0,670 & 9,35 & $10^{-3}$ & 0,237 & $\begin{array}{l}3,44 \\
0,192\end{array}$ \\
\hline Sodium Urine, $\mathrm{mM} / \mathrm{L}$ & $\begin{array}{l}119 \\
5\end{array}$ & $\begin{array}{l}114 \\
8\end{array}$ & $\begin{array}{l}89 \\
7\end{array}$ & 0,031 & 0,689 & 8,57 & 0,001 & 0,010 & $\begin{array}{l}110 \\
0,211\end{array}$ \\
\hline $\begin{array}{l}\text { Microbian Count } \\
\text { for St. aur., B/Ph }\end{array}$ & $\begin{array}{l}62,8 \\
1,2 \\
\end{array}$ & $\begin{array}{l}66,0 \\
2,0 \\
\end{array}$ & $\begin{array}{l}60,2 \\
2,3 \\
\end{array}$ & 0,024 & 0,883 & 2,52 & 0,094 & 0,624 & $\begin{array}{ll}61,6 \\
0,160 \\
\end{array}$ \\
\hline $\begin{array}{l}\text { Glucose Plasma, } \\
\mathrm{mM} / \mathrm{L}\end{array}$ & $\begin{array}{l}4,77 \\
0,17 \\
\end{array}$ & $\begin{array}{l}4,68 \\
0,33 \\
\end{array}$ & $\begin{array}{l}4,59 \\
0,18 \\
\end{array}$ & 0,027 & 0,807 & 4,55 & 0,017 & 0,532 & $\begin{array}{l}4,70 \\
0,160 \\
\end{array}$ \\
\hline $\begin{array}{l}\text { Chloride Urine } \\
\mathrm{mM} / \mathrm{L}\end{array}$ & $\begin{array}{l}102 \\
3 \\
\end{array}$ & $\begin{array}{l}127 \\
14 \\
\end{array}$ & $\begin{array}{l}96 \\
10 \\
\end{array}$ & 0,026 & 0,840 & 3,63 & 0,036 & 0,027 & $\begin{array}{l}120 \\
0,172 \\
\end{array}$ \\
\hline $\begin{array}{l}\text { Sodium Excretion, } \\
\mathrm{mM} / 24 \mathrm{~h}\end{array}$ & $\begin{array}{l}225 \\
18\end{array}$ & $\begin{array}{l}179 \\
11\end{array}$ & $\begin{array}{l}238 \\
19\end{array}$ & 0,029 & 0,743 & 6,56 & 0,004 & 0,014 & $\begin{array}{l}154 \\
0,211\end{array}$ \\
\hline $\begin{array}{l}(\mathrm{Ca} / \mathrm{K})^{0,5} \text { as Symp- } \\
\text { Vagal balance }\end{array}$ & $\begin{array}{l}0,728 \\
0,012\end{array}$ & $\begin{array}{l}0,729 \\
0,014\end{array}$ & $\begin{array}{l}0,708 \\
0,010\end{array}$ & 0,023 & 0,928 & 1,48 & 0,240 & 0,194 & $\begin{array}{l}0,710 \\
0,104\end{array}$ \\
\hline $\begin{array}{l}\text { VLF HRV PS, } \\
\text { msec }^{2}\end{array}$ & $\begin{array}{l}969 \\
99\end{array}$ & $\begin{array}{l}869 \\
141\end{array}$ & $\begin{array}{l}1238 \\
168\end{array}$ & 0,025 & 0,860 & 3,09 & 0,057 & 0,319 & $\begin{array}{l}1250 \\
0,572\end{array}$ \\
\hline $\begin{array}{l}\text { HF HRV PS, } \\
\text { msec }^{2}\end{array}$ & $\begin{array}{l}354 \\
75\end{array}$ & $\begin{array}{l}407 \\
262\end{array}$ & $\begin{array}{l}541 \\
100\end{array}$ & 0,024 & 0,900 & 2,12 & 0,134 & 0,206 & $\begin{array}{l}350 \\
0,713\end{array}$ \\
\hline $\begin{array}{l}\text { Magnesium Excre- } \\
\text { tion, } \mathrm{mM} / 24 \mathrm{~h}\end{array}$ & $\begin{array}{l}4,40 \\
0,29\end{array}$ & $\begin{array}{l}3,43 \\
0,36\end{array}$ & $\begin{array}{l}5,98 \\
0,43\end{array}$ & 0,031 & 0,703 & 8,04 & 0,001 & 0,035 & $\begin{array}{l}4,10 \\
0,256\end{array}$ \\
\hline $\begin{array}{l}\text { Lithogenicity } \\
\text { Urine }\end{array}$ & $\begin{array}{l}0,86 \\
0,03\end{array}$ & $\begin{array}{l}0,83 \\
0,03\end{array}$ & $\begin{array}{l}0,95 \\
0,03\end{array}$ & 0,028 & 0,770 & 5,67 & 0,007 & 0,443 & $\begin{array}{l}0,73 \\
0,300\end{array}$ \\
\hline $\begin{array}{l}\text { Killing Index vs } \\
\text { Staph. aur., \% }\end{array}$ & $\begin{array}{l}48,2 \\
1,5\end{array}$ & $\begin{array}{l}45,2 \\
1,9\end{array}$ & $\begin{array}{l}57,7 \\
1,4\end{array}$ & 0,026 & 0,833 & 3,81 & 0,031 & 0,375 & $\begin{array}{l}58,9 \\
0,142\end{array}$ \\
\hline $\begin{array}{l}\mathrm{CD3}^{+} \text {active } \mathrm{T}- \\
\text { Lymphocytes, \% }\end{array}$ & $\begin{array}{l}28,3 \\
0,8 \\
\end{array}$ & $\begin{array}{l}31,3 \\
0,9 \\
\end{array}$ & $\begin{array}{l}26,1 \\
1,1 \\
\end{array}$ & 0,026 & 0,817 & 4,27 & 0,021 & 0,470 & $\begin{array}{l}30,0 \\
0,167 \\
\end{array}$ \\
\hline $\begin{array}{l}\text { Interleukin-1, } \\
\text { ng/L }\end{array}$ & $\begin{array}{l}4,94 \\
0,19 \\
\end{array}$ & $\begin{array}{l}4,36 \\
0,37 \\
\end{array}$ & $\begin{array}{l}5,17 \\
0,30 \\
\end{array}$ & 0,022 & 0,964 & 0,72 & 0,495 & 0,613 & $\begin{array}{l}4,51 \\
0,173 \\
\end{array}$ \\
\hline $\begin{array}{l}\text { Potassium Urine, } \\
\mathrm{mM} / \mathrm{L}\end{array}$ & $\begin{array}{l}39,5 \\
3,2\end{array}$ & $\begin{array}{l}41,5 \\
3,6\end{array}$ & $\begin{array}{l}30,5 \\
1,7\end{array}$ & 0,026 & 0,827 & 3,99 & 0,027 & 0,022 & $\begin{array}{l}46,4 \\
0,269\end{array}$ \\
\hline $\begin{array}{l}\text { Aldosterone, } \\
\mathrm{pM} / \mathrm{L}\end{array}$ & $\begin{array}{l}225 \\
5\end{array}$ & $\begin{array}{l}236 \\
10\end{array}$ & $\begin{array}{l}229 \\
4\end{array}$ & 0,025 & 0,861 & 3,06 & 0,058 & 0,023 & $\begin{array}{l}238 \\
0,187\end{array}$ \\
\hline $\begin{array}{l}\text { ULF HRV PS, } \\
\mathrm{msec}^{2}\end{array}$ & $\begin{array}{l}73 \\
15\end{array}$ & $\begin{array}{l}139 \\
56\end{array}$ & $\begin{array}{l}110 \\
34\end{array}$ & 0,024 & 0,908 & 1,92 & 0,161 & 0,331 & $\begin{array}{l}122 \\
0,892\end{array}$ \\
\hline $\begin{array}{l}\text { HD Cholesterol } \\
\text { Plasma, mM/L }\end{array}$ & $\begin{array}{l}1,35 \\
0,08 \\
\end{array}$ & $\begin{array}{l}1,41 \\
0,14 \\
\end{array}$ & $\begin{array}{l}1,31 \\
0,08 \\
\end{array}$ & 0,023 & 0,949 & 1,02 & 0,370 & 0,458 & $\begin{array}{l}1,34 \\
0,300 \\
\end{array}$ \\
\hline
\end{tabular}

Note. In each column, the first line is the average, the second - SE or Cv. 
A number of variables, primarily cholecystokinetic activity to the standard stimulus, despite their recognizable properties, were outside the discriminant model, apparently due to duplication and/or redundancy of information (Table 2).

Table 4.2. Metabolic and neuroendocrine-immune complex parameters not included in the model

\begin{tabular}{|c|c|c|c|c|c|c|c|c|c|}
\hline \multirow[b]{2}{*}{ Variables } & \multicolumn{3}{|c|}{ Groups (n) and Means \pm SE } & \multicolumn{5}{|c|}{ Parameters of Wilks' Statistics } & \multirow[b]{2}{*}{$\begin{array}{l}\text { Norm } \\
\mathrm{Cv} \\
(30)\end{array}$} \\
\hline & $\begin{array}{l}\text { Before } \\
\text { thera- } \\
\text { py (34) }\end{array}$ & $\begin{array}{l}\text { After } \\
\text { Naftus- } \\
\text { sya (12) }\end{array}$ & $\begin{array}{l}\text { After Salt } \\
\text { Waters } \\
\text { and } N(22)\end{array}$ & $\begin{array}{l}\text { Wil } \\
\mathrm{ks} \\
\Lambda\end{array}$ & $\begin{array}{l}\text { Par- } \\
\text { tial } \\
\Lambda\end{array}$ & $\begin{array}{l}\text { F to } \\
\text { en- } \\
\text { ter }\end{array}$ & $\begin{array}{l}\text { p- } \\
\text { level }\end{array}$ & $\begin{array}{l}\text { Tole- } \\
\text { rancy }\end{array}$ & \\
\hline $\begin{array}{l}\text { Cholecystokinetic } \\
\text { Activity, units } \\
\end{array}$ & $\begin{array}{l}553 \\
22 \\
\end{array}$ & $\begin{array}{l}584 \\
24 \\
\end{array}$ & $\begin{array}{l}675 \\
28 \\
\end{array}$ & 0,021 & 0,982 & 0,34 & 0,715 &, 572 & $\begin{array}{l}624 \\
0,131 \\
\end{array}$ \\
\hline $\begin{array}{l}\text { Calcium Urine, } \\
\mathrm{mM} / \mathrm{L}\end{array}$ & $\begin{array}{l}2,34 \\
0,18\end{array}$ & $\begin{array}{l}2,40 \\
0,82\end{array}$ & $\begin{array}{l}3,04 \\
0,26\end{array}$ & 0,022 & 0,995 & 0,10 & 0,910 & 0,354 & $\begin{array}{l}3,13 \\
0,214\end{array}$ \\
\hline $\begin{array}{l}\text { Phosphates Urine, } \\
\mathrm{mM} / \mathrm{L}\end{array}$ & $\begin{array}{l}10,8 \\
0,7\end{array}$ & $\begin{array}{l}10,5 \\
1,1\end{array}$ & $\begin{array}{l}15,8 \\
1,3\end{array}$ & 0,021 & 0,975 & 0,47 & 0,629 & 0,071 & $\begin{array}{l}18,0 \\
0,294\end{array}$ \\
\hline $\begin{array}{l}\text { Potassium Plasma, } \\
\mathrm{mM} / \mathrm{L}\end{array}$ & $\begin{array}{l}4,21 \\
0,10\end{array}$ & $\begin{array}{l}4,25 \\
0,16\end{array}$ & $\begin{array}{l}4,43 \\
0,10\end{array}$ & 0,021 & 0,990 & 0,19 & 0,831 & 0,457 & $\begin{array}{l}4,55 \\
0,104\end{array}$ \\
\hline $\begin{array}{l}\text { Uric Acid Urine, } \\
\mathrm{mM} / \mathrm{L}\end{array}$ & $\begin{array}{l}2,33 \\
0,23\end{array}$ & $\begin{array}{l}1,93 \\
0,10\end{array}$ & $\begin{array}{l}1,7 \\
0,1\end{array}$ & 0,021 & 979 & 0,39 & 0,681 & 344 & $\begin{array}{l}2,14 \\
0,250\end{array}$ \\
\hline $\begin{array}{l}\text { VLF HRV PS, } \\
\%\end{array}$ & $\begin{array}{l}50,8 \\
3,0\end{array}$ & $\begin{array}{l}51,1 \\
6,5\end{array}$ & $\begin{array}{l}44,4 \\
2,5\end{array}$ & 0,021 & 0,980 & 0,37 & 0,691 & 0,346 & $\begin{array}{l}53,9 \\
0,277\end{array}$ \\
\hline $\begin{array}{l}\text { Triiodothyronine, } \\
\mathrm{nM} / \mathrm{L}\end{array}$ & $\begin{array}{l}1,97 \\
0,13\end{array}$ & $\begin{array}{l}1,93 \\
0,30\end{array}$ & $\begin{array}{l}1,78 \\
0,13\end{array}$ & 0,022 & 0,996 & 0,07 & 0,932 & 142 & $\begin{array}{l}2,20 \\
0,227\end{array}$ \\
\hline $\begin{array}{l}\text { Chloride Plasma, } \\
\mathrm{mM} / \mathrm{L}\end{array}$ & $\begin{array}{l}100,8 \\
1,0\end{array}$ & $\begin{array}{l}105,3 \\
1,2\end{array}$ & $\begin{array}{l}101,3 \\
1,6\end{array}$ & 0,022 & 0,999 & 0,02 & 0,980 & 044 & $\begin{array}{l}101,5 \\
0,032\end{array}$ \\
\hline 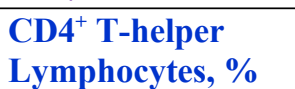 & $\begin{array}{l}28,0 \\
1,3\end{array}$ & $\begin{array}{l}34,8 \\
2,1\end{array}$ & $\begin{array}{l}26,7 \\
0,9\end{array}$ & 0,022 & 0,999 & 0,02 & 0,980 & 0,044 & $\begin{array}{l}39,5 \\
0,082\end{array}$ \\
\hline $\begin{array}{l}\text { cytolytic } \\
\text { cytes, \% }\end{array}$ & $\begin{array}{l}22,6 \\
0,8\end{array}$ & $\begin{array}{l}24,3 \\
1,5\end{array}$ & & 0,021 & 0,974 & 0,49 & 0,613 & 0,057 & $\begin{array}{l}23,5 \\
0,138\end{array}$ \\
\hline $\begin{array}{l}\text { VLD Cholesterol } \\
\text { Plasma, } \mathrm{mM} / \mathrm{L}\end{array}$ & $\begin{array}{l}0,57 \\
0,05\end{array}$ & $\begin{array}{l}0,48 \\
0,08\end{array}$ & 0,64 & 0,022 & 0,999 & 0,02 & 0,980 & 0,474 & $\begin{array}{l}0,54 \\
0,612\end{array}$ \\
\hline $\begin{array}{l}\text { LF HRV PS, } \\
\text { msec }^{2}\end{array}$ & $\begin{array}{l}717 \\
101\end{array}$ & $\begin{array}{l}691 \\
213\end{array}$ & 1604 & 0,021 & 0,965 & 0,67 & 0,519 & 0,189 & $\begin{array}{l}625 \\
0,482\end{array}$ \\
\hline $\begin{array}{l}\text { Calcium Plasma, } \\
\mathrm{mM} / \mathrm{L}\end{array}$ & $\begin{array}{l}2,20 \\
0,04\end{array}$ & $\begin{array}{l}2,23 \\
0,04\end{array}$ & $\begin{array}{l}2,2 \\
0,0 \\
\end{array}$ & 0,022 & 97 & 0,06 & 0,943 & 169 & $\begin{array}{l}2,30 \\
0,065\end{array}$ \\
\hline $\begin{array}{l}\text { Urea Plasma, } \\
\mathrm{mM} / \mathrm{L}\end{array}$ & $\begin{array}{l}5,60 \\
0,17\end{array}$ & $\begin{array}{l}6,17 \\
0,19\end{array}$ & $\begin{array}{l}6,04 \\
0,26\end{array}$ & 0,021 & 0,984 & 0,31 & 0,737 & 0,442 & $\begin{array}{l}5,00 \\
0,330\end{array}$ \\
\hline $\begin{array}{l}\text { Creatinine Urine, } \\
\mathrm{mM} / \mathrm{L}\end{array}$ & $\begin{array}{l}3,9 \\
0,3\end{array}$ & $\begin{array}{l}5,2 \\
0,6\end{array}$ & $\begin{array}{l}3,1 \\
0,3\end{array}$ & 0,021 & 0,969 & 0,60 & 0,554 & 0,246 & $\begin{array}{l}7,9 \\
0,300\end{array}$ \\
\hline $\begin{array}{l}\text { Cortisol, } \\
\text { nM/L }\end{array}$ & $\begin{array}{l}373 \\
26\end{array}$ & $\begin{array}{l}441 \\
30\end{array}$ & 419 & 0,021 & 0,993 & 0,13 & 0,875 & 0,716 & $\begin{array}{l}405 \\
0,524\end{array}$ \\
\hline $\begin{array}{l}\text { Parathyroid } \\
\text { activity, units }\end{array}$ & $\begin{array}{l}1,81 \\
0,06\end{array}$ & $\begin{array}{l}1,73 \\
0,07\end{array}$ & $\begin{array}{l}1,90 \\
0,04\end{array}$ & 0,022 & 0,999 & 0,37 & 0,519 & 0,189 & $\begin{array}{l}1,82 \\
0,230\end{array}$ \\
\hline $\begin{array}{l}\text { Blood Pressure } \\
\text { systolic, mmHg }\end{array}$ & $\begin{array}{l}141,2 \\
2,7\end{array}$ & $\begin{array}{l}141,9 \\
5,3\end{array}$ & $\begin{array}{l}141,4 \\
3,5\end{array}$ & 0,021 & 0,978 & 0,42 & 0,663 & 0,493 & $\begin{array}{l}124,5 \\
0,076\end{array}$ \\
\hline $\begin{array}{l}\text { Blood Pressure } \\
\text { diastolic, mmHg }\end{array}$ & $\begin{array}{l}84,6 \\
1,7\end{array}$ & $\begin{array}{l}85,7 \\
1,7\end{array}$ & $\begin{array}{l}86,0 \\
8,2\end{array}$ & 0,021 & 0,987 & 0,25 & 0,780 & 0,476 & $\begin{array}{l}79,0 \\
0,054\end{array}$ \\
\hline $\begin{array}{l}\text { Kerdoe Vegetativ } \\
\text { Index, units }\end{array}$ & $\begin{array}{l}-18,8 \\
3,4\end{array}$ & $\begin{array}{l}-23,1 \\
5,1\end{array}$ & $\begin{array}{l}-19,5 \\
3,8\end{array}$ & 0,021 & 0,969 & 0,13 & 0,663 & 0,442 & $\begin{array}{l}-23,5 \\
20,1\end{array}$ \\
\hline
\end{tabular}


Table 3. Summary of step-by-step analysis of discriminant variables ranked by criterion $\Lambda$

\begin{tabular}{|c|c|c|c|c|c|}
\hline Variables currently in the model & $\begin{array}{l}\mathrm{F} \text { to } \\
\text { enter }\end{array}$ & $\begin{array}{l}\text { p- } \\
\text { level }\end{array}$ & $\Lambda$ & $\begin{array}{l}\text { F-va- } \\
\text { lue }\end{array}$ & $\begin{array}{l}\mathrm{p}- \\
\text { level }\end{array}$ \\
\hline Phosphates Excretion, mM/24 h & 33,0 & $10^{-6}$ & 0,496 & 33,0 & $10^{-6}$ \\
\hline Calcitonin, ng/L & 7,42 & 0,001 & 0,403 & 18,4 & $10^{-6}$ \\
\hline Creatinine Plasma, $\mu \mathrm{M} / \mathrm{L}$ & 5,95 & 0,004 & 0,279 & 13,8 & $10^{-6}$ \\
\hline Testosterone, $\mathrm{nM} / \mathrm{L}$ & 5,86 & 0,005 & 0,234 & 13,0 & $10^{-6}$ \\
\hline Sodium Plasma, mM/L & 5,68 & 0,005 & 0,197 & 12,5 & $10^{-6}$ \\
\hline Phosphate Plasma, mM/L & 5,55 & 0,006 & 0,166 & 12,3 & $10^{-6}$ \\
\hline Magnesium Urine, mM/L & 5,20 & 0,008 & 0,140 & 12,1 & $10^{-6}$ \\
\hline Chloride Excretion, $\mathrm{mM} / 24 \mathrm{~h}$ & 4,11 & 0,022 & 0,123 & 11,7 & $10^{-6}$ \\
\hline Interleukin-6, ng/L & 3,38 & 0,041 & 0,110 & 11,3 & $10^{-6}$ \\
\hline LD Cholesterol Plasma, $\mathrm{mM} / \mathrm{L}$ & 3,83 & 0,028 & 0,096 & 11,1 & $10^{-6}$ \\
\hline Sodium Urine, $\mathrm{mM} / \mathrm{L}$ & 2,83 & 0,068 & 0,087 & 10,8 & $10^{-6}$ \\
\hline Microbian Count for Staph. aur., Bac/Ph & 2,49 & 0,092 & 0,080 & 10,4 & $10^{-6}$ \\
\hline Glucose Plasma, mM/L & 2,44 & 0,097 & 0,073 & 10,1 & $10^{-6}$ \\
\hline Chloride Urine $\mathrm{mM} / \mathrm{L}$ & 2,51 & 0,092 & 0,066 & 9,81 & $10^{-6}$ \\
\hline Sodium Excretion, mM/24 h & 2,05 & 0,140 & 0,061 & 9,51 & $10^{-6}$ \\
\hline$(\mathrm{Ca} / \mathrm{K})^{0,5}$ Plasma as Symp/Vagal balance & 1,75 & 0,185 & 0,057 & 9,18 & $10^{-6}$ \\
\hline VLF HRV PS, msec ${ }^{2}$ & 1,40 & 0,255 & 0,054 & 8,81 & $10^{-6}$ \\
\hline HF HRV PS, msec $^{2}$ & 4,41 & 0,018 & 0,045 & 9,13 & $10^{-6}$ \\
\hline Magnesium Excretion, mM/24 h & 2,16 & 0,127 & 0,042 & 8,99 & $10^{-6}$ \\
\hline$(\mathrm{UA} \cdot \mathrm{Ca}) /(\mathrm{Cr} \bullet \mathrm{Mg})^{0,25}$ Lithogenicity Urine & 3,04 & 0,058 & 0,037 & 9,06 & $10^{-6}$ \\
\hline Killing Index vs Staph. aur., \% & 2,17 & 0,126 & 0,033 & 8,96 & $10^{-6}$ \\
\hline $\mathrm{CD3}^{+}$active T-Lymphocytes, \% & 2,33 & 0,109 & 0,030 & 8,92 & $10^{-6}$ \\
\hline Interleukin-1, ng/L & 1,10 & 0,341 & 0,029 & 8,61 & $10^{-6}$ \\
\hline Potassium Urine, $\mathrm{mM} / \mathrm{L}$ & 1,17 & 0,321 & 0,027 & 8,34 & $10^{-6}$ \\
\hline Aldosterone, pM/L & 1,54 & 0,226 & 0,025 & 8,18 & $10^{-6}$ \\
\hline ULF HRV PS, msec $^{2}$ & 1,95 & 0,155 & 0,023 & 8,12 & $10^{-6}$ \\
\hline HD Cholesterol Plasma, mM/L & 1,02 & 0,370 & 0,022 & 7,87 & $10^{-6}$ \\
\hline
\end{tabular}

The identifying information contained in the 27 discriminant variables is condensed into two roots. The major root contains $80 \%$ of discriminatory opportunities $\left(r^{*}=0,958\right.$; Wilks' $\left.\Lambda=0,022 ; \chi_{(56)}^{2}=197 ; \mathrm{p}<10^{-6}\right)$, while minor root $-20 \%$ only $\left(\mathrm{r}^{*}=0,857\right.$; Wilks' $\Lambda=0,265$; $\left.\chi_{(27)}^{2}=68 ; \mathrm{p}<10^{-4}\right)$.

Calculating the values of discriminant roots for each patient as the sum of the products of non-standardized (raw) coefficients for individual values of discriminant variables together with the constant (Table 4) allows visualization of each patient in the information space of roots (Fig. 1). 
Table 4. Standardized and raw coefficients and constants for discriminant variables

\begin{tabular}{|c|c|c|c|c|}
\hline Coefficients & \multicolumn{2}{|c|}{ Standardized } & \multicolumn{2}{|c|}{ Raw } \\
\hline Variables & Root 1 & Root 2 & Root 1 & Root 2 \\
\hline Phosphates Excretion, mM/24 h & $-1,619$ & 0,244 & $-0,1389$ & 0,0210 \\
\hline Calcitonin, ng/L & $-0,802$ & 0,038 & $-0,1817$ & 0,0085 \\
\hline Creatinine Plasma, $\mu \mathrm{M} / \mathrm{L}$ & 0,857 & $-0,435$ & 0,0678 & $-0,0344$ \\
\hline Testosterone, $\mathrm{nM} / \mathrm{L}$ & 0,389 & $-1,150$ & 0,0448 & $-0,1325$ \\
\hline Sodium Plasma, mM/L & $-0,434$ & 1,159 & $-0,0493$ & 0,1318 \\
\hline Phosphate Plasma, mM/L & $-0,0586$ & 1,031 & $-0,3130$ & 5,5092 \\
\hline Magnesium Urine, mM/L & $-1,217$ & $-0,886$ & $-1,8362$ & $-1,3358$ \\
\hline Chloride Excretion, $\mathrm{mM} / 24 \mathrm{~h}$ & 0,666 & $-1,712$ & 0,0071 & $-0,0182$ \\
\hline Interleukin-6, ng/L & $-0,161$ & $-0,926$ & $-0,0841$ & $-0,4839$ \\
\hline LD Cholesterol Plasma, $\mathrm{mM} / \mathrm{L}$ & 1,059 & 0,701 & 1,0159 & 0,6727 \\
\hline Sodium Urine, $\mathrm{mM} / \mathrm{L}$ & 5,833 & $-0,777$ & 0,1913 & $-0,0255$ \\
\hline Microbian Count for Staph. aur., Bac/Ph & 0,4101 & 0,2130 & 0,0491 & 0,0255 \\
\hline Glucose Plasma, mM/L & $-0,502$ & 0,423 & $-0,5240$ & 0,4415 \\
\hline Chloride Urine $\mathrm{mM} / \mathrm{L}$ & $-1,351$ & 2,433 & $-0,0379$ & 0,0683 \\
\hline Sodium Excretion, mM/24 h & $-4,335$ & 1,370 & $-0,0463$ & 0,0146 \\
\hline$(\mathrm{Ca} / \mathrm{K})^{0,5}$ Plasma as Symp/Vagal balance & $-0,566$ & 0,327 & $-9,7651$ & 5,6385 \\
\hline VLF HRV PS, msec ${ }^{2}$ & $-0,440$ & 0,596 & $-0,0007$ & 0,0009 \\
\hline HF HRV PS, msec $^{2}$ & 0,596 & $-0,468$ & 0,0011 & $-0,0008$ \\
\hline Magnesium Excretion, $\mathrm{mM} / 24 \mathrm{~h}$ & 2,973 & 0,751 & 1,7012 & 0,4299 \\
\hline$(\mathrm{UA} \cdot \mathrm{Ca}) /(\mathrm{Cr} \bullet \mathrm{Mg})^{0,25}$ Lithogenicity Urine & $-0,714$ & $-0,262$ & $-5,0313$ & $-1,8465$ \\
\hline Killing Index vs Staph. aur., \% & $-0,624$ & $-0,346$ & $-0,0821$ & $-0,0455$ \\
\hline $\mathrm{CD3}^{+}$active T-Lymphocytes, \% & 0,325 & 0,632 & 0,0700 & 0,1363 \\
\hline Interleukin-1, ng/L & $-0,191$ & 0,187 & $-0,1547$ & 0,1510 \\
\hline Potassium Urine, $\mathrm{mM} / \mathrm{L}$ & $-2,663$ & $-1,391$ & $-0,1784$ & $-0,0932$ \\
\hline Aldosterone, pM/L & 2,345 & 1,221 & 0,0876 & 0,0456 \\
\hline ULF HRV PS, msec $^{2}$ & $-0,3119$ & $-0,5054$ & $-0,0023$ & $-0,0037$ \\
\hline \multirow[t]{3}{*}{ HD Cholesterol Plasma, mM/L } & 0,344 & 0,057 & 0,7764 & 0,1275 \\
\hline & \multicolumn{2}{|c|}{ Constants } & $-10,23$ & $-37,42$ \\
\hline & \multicolumn{2}{|c|}{ Eigenvalues } & 11,26 & 2,77 \\
\hline \multicolumn{3}{|c|}{ Cumulative Proportion } & 0,802 & 1 \\
\hline
\end{tabular}

Following the accepted algorithm, Table 5 collects the Z-scores of discriminant variables together with those that are not included in the model, but still reflect the specifics of the water used. 
Table 5. Correlations between immune variables and roots, centroids of clusters and Zscores of clusters

\begin{tabular}{|c|c|c|c|c|c|}
\hline \multirow{2}{*}{$\begin{array}{l}\text { Variables } \\
\text { Root } 1(80 \%)\end{array}$} & \multicolumn{2}{|c|}{$\begin{array}{c}\text { Correlations } \\
\text { Variables-Roots }\end{array}$} & \multirow{2}{*}{$\begin{array}{c}\text { After } \\
\text { Salt Waters } \\
\text { and N (22) } \\
-4,73\end{array}$} & \multirow{2}{*}{$\begin{array}{c}\begin{array}{c}\text { After } \\
\text { Naftussya } \\
(\mathbf{1 2})\end{array} \\
+1,87\end{array}$} & \multirow{2}{*}{$\begin{array}{c}\begin{array}{c}\text { Before } \\
\text { therapy } \\
(44)\end{array} \\
+2,40\end{array}$} \\
\hline & Root 1 & Root 2 & & & \\
\hline Phosphates Excretion & $-0,299$ & $-0,063$ & $+2,31$ & $-1,14$ & $-0,94$ \\
\hline Magnesium Excretion & $-0,146$ & $-0,141$ & $+1,79$ & $-0,64$ & $+0,29$ \\
\hline Chloride Excretion & $-0,106$ & 0,012 & $+3,16$ & $+1,02$ & $+0,62$ \\
\hline$(\mathrm{UA} \cdot \mathrm{Ca}) /(\mathrm{Cr} \cdot \mathrm{Mg})^{0,25}$ Lithogenicity Urine & $-0,094$ & $-0,066$ & $+0,98$ & $+0,43$ & $+0,59$ \\
\hline HF HRV PS & $-0,045$ & 0,016 & $+0,82$ & $-0,03$ & $-0,04$ \\
\hline Cholecystokinetic Activity & & & $+0,62$ & $-0,30$ & $-0,86$ \\
\hline Killing Index vs Staph. aureus & $-0,190$ & $-0,111$ & $-0,15$ & $-1,64$ & $-1,28$ \\
\hline Potassium Plasma & & & $-0,25$ & $-0,64$ & $-0,72$ \\
\hline Calcium Urine & & & $-0,13$ & $-1,09$ & $-1,18$ \\
\hline Phosphates Urine & & & $-0,42$ & $-1,36$ & $-1,41$ \\
\hline Triiodothyronine & & & $-0,46$ & $-0,55$ & $-0,85$ \\
\hline VLF HRV PS ${ }^{2}$ & $-0,065$ & $-0,043$ & $-0,01$ & $-0,47$ & $-0,36$ \\
\hline Calcitonin & $-0,119$ & $-0,054$ & $-0,51$ & $-1,14$ & $-1,02$ \\
\hline Phosphate Plasma & 0,108 & 0,122 & $-1,43$ & $-0,36$ & $-0,82$ \\
\hline Sodium Urine & 0,135 & $-0,022$ & $-0,90$ & $+0,17$ & $+0,39$ \\
\hline Uric Acid Urine & & & $-0,65$ & $-0,24$ & $+0,30$ \\
\hline Potassium Urine & 0,089 & 0,041 & $-1,27$ & $-0,40$ & $-0,56$ \\
\hline LD Cholesterol Plasma & 0,036 & $-0,019$ & $-0,28$ & $-0,11$ & $+0,16$ \\
\hline Glucose Plasma & 0,025 & $-0,018$ & $-0,15$ & $-0,02$ & $+0,09$ \\
\hline$(\mathrm{Ca} / \mathrm{K})^{0,5}$ Plasma as Symp/Vagal balance & 0,051 & 0,009 & $-0,05$ & $+0,23$ & $+0,24$ \\
\hline Root $2(20 \%)$ & Root 1 & Root 2 & $-0,14$ & $+3,39$ & $-1,11$ \\
\hline Testosterone & 0,019 & $-0,241$ & $+0,24$ & $-0,82$ & $+0,84$ \\
\hline Magnesium Urine & 0,027 & $-0,082$ & $-0,95$ & $-1,05$ & $-0,71$ \\
\hline Interleukin-6 & $-0,022$ & $-0,093$ & $+0,24$ & $-0,42$ & $+0,14$ \\
\hline Interleukin-1 & $-0,040$ & $-0,110$ & $+0,78$ & $-0,24$ & $+0,50$ \\
\hline VLD Cholesterol Plasma & & & $+0,09$ & $-0,21$ & $+0,32$ \\
\hline Parathyroid activity & & & $+0,19$ & $-0,22$ & $-0,03$ \\
\hline Creatinine Plasma & 0,032 & $-0,185$ & $+0,60$ & $+0,18$ & $+0,99$ \\
\hline Urea Plasma & & & $+0,63$ & $+0,45$ & $+0,83$ \\
\hline Sodium Excretion & $-0,035$ & $-0,111$ & $+2,58$ & $+0,78$ & $+2,17$ \\
\hline Chloride Urine & 0,047 & 0,164 & $-1,15$ & $+0,38$ & $-0,85$ \\
\hline Creatinine Urine & & & $-2,02$ & $-1,11$ & $-1,69$ \\
\hline Chloride Plasma & & & $-0,07$ & $+1,00$ & $-0,26$ \\
\hline $\mathrm{CD3}^{+}$active T-Lymphocytes & 0,088 & 0,151 & $-0,78$ & $+0,25$ & $-0,33$ \\
\hline Sodium Plasma & 0,005 & 0,130 & $-0,55$ & $+0,33$ & $-0,71$ \\
\hline Calcium Plasma & & & $-0,64$ & $-0,44$ & $-0,66$ \\
\hline Microbian Count for Staph. aureus & 0,056 & 0,092 & $-0,14$ & $+0,44$ & $+0,12$ \\
\hline HD Cholesterol Plasma & 0,017 & 0,031 & $-0,08$ & $+0,21$ & $+0,04$ \\
\hline ULF HRV PS & $-0,023$ & 0,103 & $-0,11$ & $+0,16$ & $-0,45$ \\
\hline Aldosterone & $-0,012$ & 0,091 & $-0,19$ & $-0,05$ & $-0,30$ \\
\hline
\end{tabular}

The localization in the extreme left zone of the axis of the first root of the cluster of patients who received two mineral waters shows a significant increase relative to baseline levels of parameters that are negatively associated with the root, and a significant decrease in positively correlated with the root parameters. In contrast, in patients receiving Naftussya water only, these parameters remained unchanged or changed to a much lesser extent.

On the other hand, such patients are characterized by a significant decrease/increase in another number of parameters associated with the second root negatively/positively, while in combination balneotherapy their changes are insignificant or much less pronounced. 


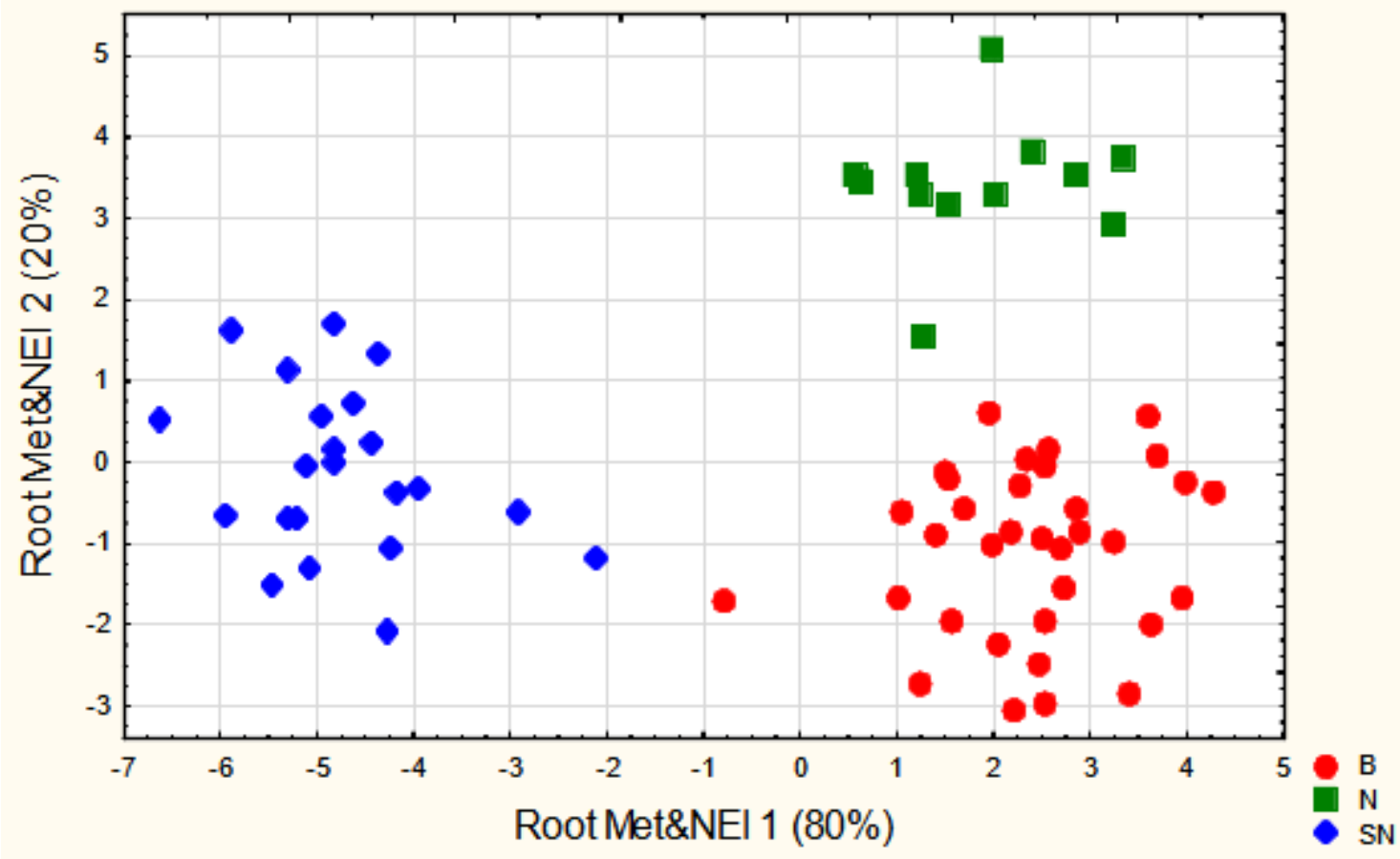

Fig. 1. Scattering of individual values of the first and second discriminant roots of patients before (circles) and after the course of drinking only water Naftussya (squares) and in combination with water "Myroslava" or "Khrystyna" (rhombuses)

Fig. 2 illustrates that the integrated initial state of all three groups of patients was almost the same as the effect on the discriminant variables of both sulfate-chloride sodiummagnesium mineral waters.

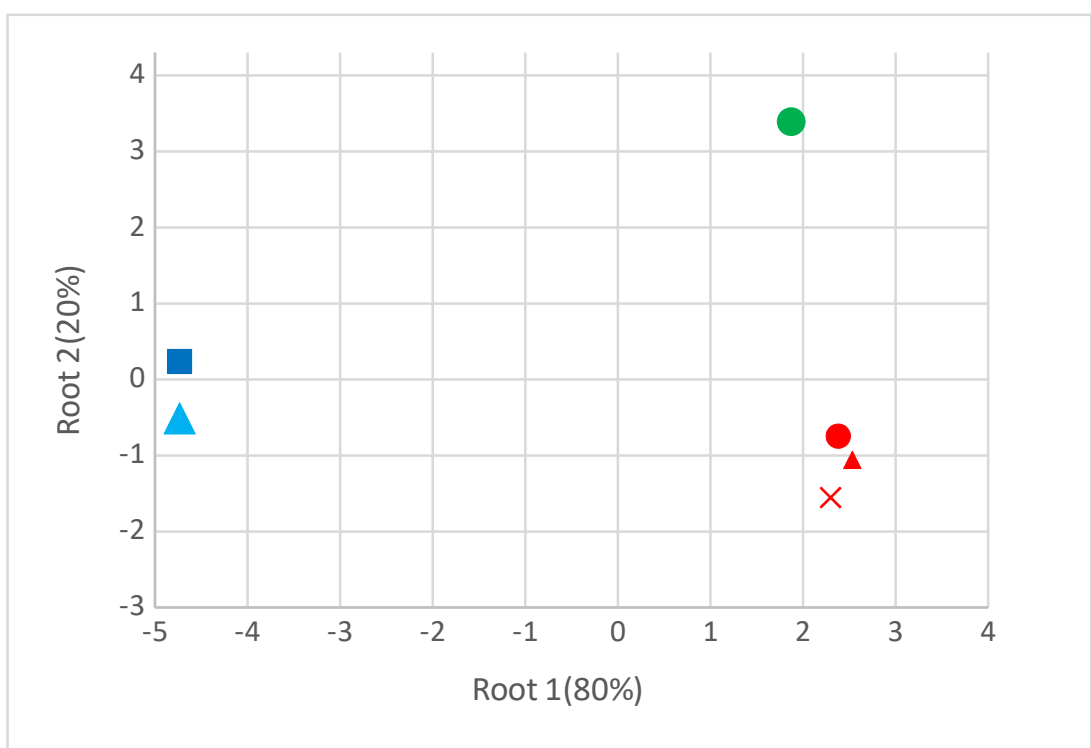

Fig. 2. Mean values $(M \pm S D)$ of the first and second discriminant roots of patients before (red fill) and after the course of drinking only water "Naftussya" (circle) and in combination with water "Myroslava" (triangle) or "Khrystyna" (square)

The visual impression of a clear demarcation of the three clusters in the information field of the two roots is documented by calculating the distances of Mahalanobis (Table 6). 
Table 6. Squares of Mahalanobis distances between clusters (above the diagonal) and Fcriteria $(\mathrm{d} f=15.4)$ and $p$-levels (below the diagonal)

\begin{tabular}{|l|l|l|l|}
\hline Clusters & $\begin{array}{l}\text { Before } \\
\text { therapy }\end{array}$ & $\begin{array}{l}\text { After } \\
\text { Naftussya }\end{array}$ & $\begin{array}{l}\text { After } \\
\text { SW\&N }\end{array}$ \\
\hline $\begin{array}{l}\text { Before } \\
\text { therapy }\end{array}$ & 0 & 21 & 52 \\
\hline $\begin{array}{l}\text { After } \\
\text { Naftussy } \\
\text { a }\end{array}$ & $\begin{array}{l}3,8 \\
10^{-4}\end{array}$ & 0 & 56 \\
\hline $\begin{array}{l}\text { After } \\
\text { SW\&N }\end{array}$ & $\begin{array}{l}14,5 \\
10^{-6}\end{array}$ & $\begin{array}{l}9,1 \\
10^{-6}\end{array}$ & 0 \\
\hline
\end{tabular}

Selected discriminant variables were used to identify the affiliation of a patient to a particular cluster. This goal of discriminant analysis is realized with the help of classification functions (Table7).

Table 7. Coefficients and constants of classification functions

\begin{tabular}{|c|c|c|c|}
\hline Clusters & $\begin{array}{c}\text { Before } \\
\text { therapy }\end{array}$ & $\begin{array}{c}\text { After } \\
\text { Naftussya }\end{array}$ & $\begin{array}{c}\text { After } \\
\text { Salt W\&N } \\
\end{array}$ \\
\hline Variables & $\mathrm{p}=, 500$ & $\mathrm{p}=, 176$ & $\mathrm{p}=, 324$ \\
\hline Phosphates Excretion, mM/24 h & $-0,742$ & $-0,574$ & 0,270 \\
\hline Calcitonin, ng/L & $-2,613$ & $-2,478$ & $-1,308$ \\
\hline Creatinine Plasma, $\mu \mathrm{M} / \mathrm{L}$ & $-0,164$ & $-0,355$ & $-0,681$ \\
\hline Testosterone, nM/L & $-2,692$ & $-3,312$ & $-3,141$ \\
\hline Sodium Plasma, mM/L & 5,563 & 6,182 & 6,043 \\
\hline Phosphate Plasma, mM/L & 471,4 & 496,4 & 479,0 \\
\hline Magnesium Urine, $\mathrm{mM} / \mathrm{L}$ & $-41,67$ & $-46,71$ & $-29,86$ \\
\hline Chloride Excretion, $\mathrm{mM} / 24 \mathrm{~h}$ & $-0,108$ & $-0,194$ & $-0,176$ \\
\hline Interleukin-6, ng/L & $-30,56$ & $-32,69$ & $-30,43$ \\
\hline LD Cholesterol Plasma, mM/L & 70,60 & 73,08 & 64,00 \\
\hline Sodium Urine, mM/L & 7,063 & 6,846 & 5,673 \\
\hline Microbian Count for Staph. aur., Bac/Ph & 3,221 & 3,310 & 2,895 \\
\hline Glucose Plasma, mM/L & 12,50 & 14,76 & 16,66 \\
\hline Chloride Urine $\mathrm{mM} / \mathrm{L}$ & 0,682 & 1,009 & 1,019 \\
\hline Sodium Excretion, mM/24 h & $-0,511$ & $-0,420$ & $-0,166$ \\
\hline$(\mathrm{Ca} / \mathrm{K})^{0,5}$ Plasma as Symp/Vagal balance & 149,1 & 179,7 & 224,2 \\
\hline VLF HRV PS, msec ${ }^{2}$ & 0,091 & 0,095 & 0,097 \\
\hline HF HRV PS, msec ${ }^{2}$ & $-0,088$ & $-0,092$ & $-0,097$ \\
\hline Magnesium Excretion, $\mathrm{mM} / 24 \mathrm{~h}$ & 34,30 & 35,33 & 22,57 \\
\hline$(\mathrm{UA} \cdot \mathrm{Ca}) /(\mathrm{Cr} \bullet \mathrm{Mg})^{0,25}$ Lithogenicity Urine & 40,54 & 34,90 & 74,66 \\
\hline Killing Index vs Staph. aureus, \% & $-0,166$ & $-0,328$ & 0,376 \\
\hline $\mathrm{CD3}^{+}$active T-Lymphocytes, $\%$ & 10,05 & 10,62 & 9,678 \\
\hline Interleukin-1, ng/L & 7,842 & 8,604 & 9,093 \\
\hline Potassium Urine, $\mathrm{mM} / \mathrm{L}$ & $-19,99$ & $-20,32$ & $-18,81$ \\
\hline Aldosterone, pM/L & 10,02 & 10,18 & 9,436 \\
\hline ULF HRV PS, msec $^{2}$ & $-0,512$ & $-0,528$ & $-0,500$ \\
\hline HD Cholesterol Plasma, mM/L & 26,96 & 27,12 & 21,55 \\
\hline Constants & -2151 & -2319 & -2122 \\
\hline
\end{tabular}


The use of classification functions allows unmistakable retrospective identification of all clusters (Table 8).

Table 8. Classification matrix

Rows: observed classifications; columns: projected classifications

\begin{tabular}{|l|l|l|l|l|}
\hline & \multirow{2}{*}{$\begin{array}{l}\text { Percent } \\
\text { Correct }\end{array}$} & $\begin{array}{l}\text { Before } \\
\text { therapy }\end{array}$ & $\begin{array}{l}\text { After } \\
\text { Naftussya }\end{array}$ & $\begin{array}{l}\text { After } \\
\text { Salt W\&N }\end{array}$ \\
\cline { 3 - 5 } Groups & $\mathrm{p}=, 500$ & $\mathrm{p}=, 176$ & $\mathrm{p}=, 324$ \\
\hline $\begin{array}{l}\text { Before } \\
\text { therapy }\end{array}$ & 100 & $\mathbf{3 4}$ & 0 & 0 \\
\hline $\begin{array}{l}\text { After } \\
\text { Naftussya }\end{array}$ & 100 & 0 & $\mathbf{1 2}$ & 0 \\
\hline $\begin{array}{l}\text { After } \\
\text { Salt W\&N }\end{array}$ & 100 & 0 & 0 & $\mathbf{2 2}$ \\
\hline Total & 100 & 34 & 12 & 22 \\
\hline
\end{tabular}

Thus, we have shown that complex balneotherapy by interval use of sulfate-chloride sodium-magnesium mineral water with Naftussya water causes significant changes in the constellation of neuroendocrine, metabolic and immune parameters, which are different from the effects of Naftussya water monotherapy.

In the conditions of the resort, it was organizationally (but also ethically) impossible to offer patients to use only newly created mineral waters. However, the calculation of algebraic differences between the mean Z-scores of the parameters in both groups of patients still allows us to assess the independent effects of sulfate-chloride sodium-magnesium mineral waters.

This approach suggests that sulfate-chloride sodium-magnesium mineral waters have their own (per se) more or less pronounced effect on the constellation of parameters of the neuroendocrine-immune complex and metabolism, regardless of their initial levels (Fig. 3).

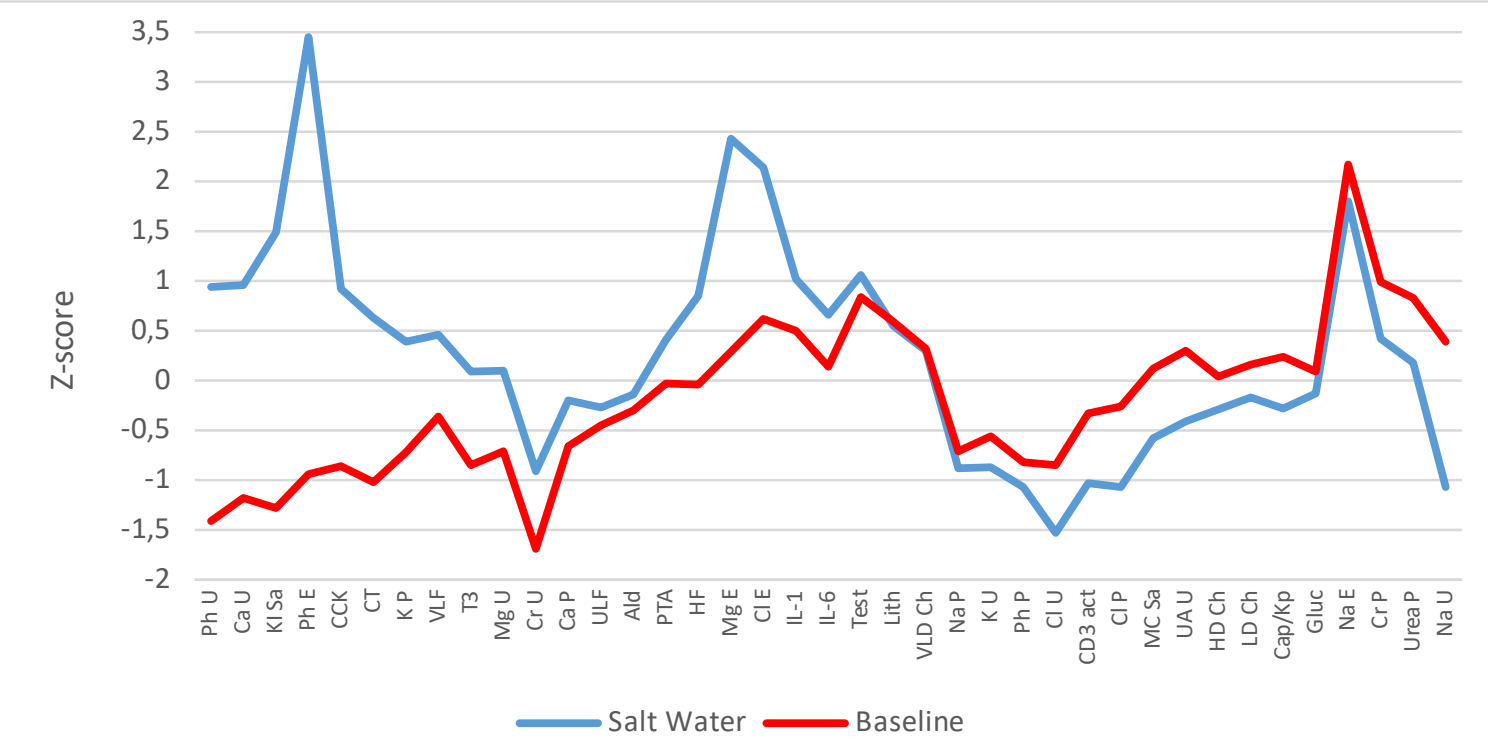

Fig. 3. Profiles of real $Z$-scores of initial discriminant variables and their simulated Z-scores after consumption of sulphate-chloride sodium-magnesium mineral waters

In particular, initially reduced neuroendocrine (VLF and ULF bands HRV, calcitonin, triiodothyronine, to a lesser extent aldosterone and parathyroid activity) and metabolic (urine 
concentrations of phosphate, calcium, magnesium and creatinine, phosphaturia, plasma potassium and calcium, cholecystokinetic activity) variables as well as the completion of phagocytosis of Staphylococcus aureus increase, as a rule, to the zone of norm. On the other hand, initially increased urinary excretion and concentration of sodium and plasma creatinine and urea levels are reduced. Such effects are consistent with the ancient concept of the ambivalent-balancing nature of the effects of balneal factors on the body [2].

However, there is an increase in initially normal levels of vagal tone, parathyroid activity, excretion of magnesium and chloride and interleukins 1 and 6 plasma, as well as a decrease in initially normal levels of $\mathrm{Ca} / \mathrm{K}$ marker sympathetic-vagal balance, concentration of uric acid in urine as well as glucose and cholesterol in plasma as well as the intensity of Staphylococcus aureus phagocytosis. The latter pattern is formed by initially reduced plasma levels of sodium, phosphate and chloride, chloride and potassium of urine, as well as active Tlymphocytes of blood, which continue to decline. Such effects do not fit into this concept, but are consistent with the known data on the diversity of responses of the neuroendocrineimmune complex and metabolism to balneal factors [14,15,17,22,25].

\section{CONCLUSION}

The newly created sulfate-chloride sodium-magnesium drinking mineral waters of Truskavets resort have favorable neuroendocrine, metabolic and immune effects on patients with chronic cholecystitis and pyelonephritis.

\section{ACKNOWLEDGMENT}

We express sincere gratitude to TA Korolyshyn, VV Kikhtan as well as administration of clinical sanatorium "Moldova" for help in recording tests.

\section{ACCORDANCE TO ETHICS STANDARDS}

Tests in patients are carried out in accordance with positions of Helsinki Declaration 1975, revised and complemented in 2002, and directive of National Committee on ethics of scientific researches. During realization of tests from all participants the informed consent is got and used all measures for providing of anonymity of participants.

\section{REFERENCES}

1. Baevsky RM, Ivanov GG. Heart Rate Variability: theoretical aspects and possibilities of clinical application [in Russian]. Ultrazvukovaya i funktsionalnaya diagnostika. 2001; 3: 106-127.

2. Balanovs'kyi VP, Popovych IL, Karpynets' SV. About ambivalence-equilibratory character of influence of curative water Naftussya on organism of human [in Ukrainian]. Dopovidi ANU. Mat pryr tekhn Nauky. 1993; 3: 154-158.

3. Berntson GG, Bigger JT jr, Eckberg DL, Grossman P, Kaufman PG, Malik M, Nagaraja HN, Porges SW, Saul JP, Stone PH, Van der Molen MW. Heart Rate Variability: Origines, methods, and interpretive caveats. Psychophysiology. 1997; 34: 623-648.

4. Douglas SD, Quie PG. Investigation of Phagocytes in Disease. Churchil; 1981: 110.

5. Fajda OI, Hrinchenko BV, Snihur OV, Barylyak LG, Zukow W. What Kerdoe's Vegetative Index really reflects? Journal of Education, Health and Sport. 2015; 5(12): 279-288.

6. Fajda OI, Drach OV, Barylyak LG, Zukow W. Relationships between $\mathrm{Ca} / \mathrm{K}$ plasma ratio and parameters of Heart Rate Variability at patients with diathesis urica. Journal of Education, Health and Sport. 2016; 6(1): 295-301.

7. Flyunt VR, Flyunt I-SS, Fil' VM, Kovbasnyuk MM, Hryvnak RF, Popel SL, Zukow W. Relationships between caused by drinking of bioactive water Naftussya changes in urine lithogenicity and neuro-humoral-immune factors in humans with their abnormalities. Journal of Education, Health and Sport. 2017; 7(3): 11-30. 
8. Goryachkovskiy AM. Clinical Biochemistry [in Russian]. Odesa: Astroprint; 1998: 608.

9. Gozhenko AI, Korda MM, Popadynets' OO, Popovych IL. Entropy, Harmony, Synchronization, Harmony and Their Neuro-Endocrine-Immune Correlates [in Ukrainian]. Odesa. Feniks; 2021: 232.

10. Heart Rate Variability. Standards of Measurement, Physiological Interpretation, and Clinical Use. Task Force of ESC and NASPE. Circulation. 1996; 93(5): 1043-1065.

11. Hrytsak MV, Barylyak LG, Usyns'kyi RS, Mysula IR. Endocrine and metabolic effects of sulfate chloride sodium-magnesium mineral waters "Myroslava" and "Khrystyna" of Truskavets' spa in healthy female rats. In: Proceedings of the XII All-Ukrainian scientific-practical conference "Topical issues of pathology under the influence of extraordinary factors on the body". Galician Readings II (Ternopil', October 29-30, 2020). Ternopil'; 2020: 125-127.

12. Hrytsak MV, Popovych DV, Badiuk NS, Hrytsan II, Zukow W. Similar neuroendocrine and metabolic effects of sulfate-chloride sodium-magnesium mineral waters "Myroslava" and "Khrystyna" of Truskavets' spa in healthy female rats. Journal of Education, Health and Sport. 2021; 11(6): 320334.

13. Hrytsak MV, Popovych DV, Badiuk NS, Hrytsan II, Zukow W. Peculiaritias of neuroendocrine and metabolic effects of sulfate-chloride sodium-magnesium mineral waters "Myroslava" and "Khrystyna" of Truskavets' spa in healthy female rats. Journal of Education, Health and Sport. 2021; 11(9): 862-875.

14. Hrytsan II, Gozhenko AI, Badiuk NS, Zukow W. Variants of the state of electrolyte exchange in female rates. In: Rehabilitation Medicine and Health-Resort Institutions Development. Proceedings of the 19th International Applied Research Conference (Kyïv, 11-12 December 2019). Edited by O. Gozhenko, W. Zukow. Toruń, Kyiv. 2019: 25-26.

15. Hrytsan II, Gozhenko AI, Badiuk NS, Zukow W. Variants of the state of electrolyte exchange in female rates. Journal of Education, Health and Sport. 2019; 9(10): 262-279.

16. Klecka WR. Discriminant Analysis [trans. from English in Russian] (Seventh Printing, 1986). In: Factor, Discriminant and Cluster Analysis. Moskva: Finansy i Statistika; 1989: 78-138.

17. Kozyavkina NV, Popovych IL, Zukow W. Metabolic accompaniment of thyrotropic effects of bioactive water Naftussya at the women with thyroid hyperplasia. Journal of Health Sciences. 2013; 3(5): 409-424.

18. Kul'chyns'kyi AB, Kovbasnyuk MM, Korolyshyn TA, Kyjenko VM, Zukow W, Popovych IL. Neuro-immune relationships at patients with chronic pyelonephrite and cholecystite. Communication 2. Correlations between parameters EEG, HRV and Phagocytosis. Journal of Education, Health and Sport. 2016; 6(10): 377-401.

19. Lapovets' LYe, Lutsyk BD. Handbook of Laboratory Immunology [in Ukrainian]. L'viv; 2004: 173 .

20. Marfiyan OM, Korolyshyn TA, Barylyak LG, Kovbasnyuk MM, Yavors'kyi OV, Zukow W, Popovych IL. Neuroendocrine-immune and metabolic accompaniments of cholecystokinetic effects of balneotherapy on spa Truskavets'. Journal of Education, Health and Sport. 2015; 5(5): 21-30.

21. Marfiyan OM, Zukow W. Relationships between parameters of gall-bladder motility and neuroendocrine-immune complex and metabolism in men with chronic cholecystitis and pyelonephritis. Journal of Education, Health and Sport. 2015; 5(12): 434-449.

22. Popovych IL, Kul'chyns'kyi AB, Korolyshyn TA, Zukow W. Interrelations between changes in parameters of HRV, EEG and cellular immunity at patients with chronic pyelonephritis and cholecystitis. Journal of Education, Health and Sport. 2017; 7(10): 11-23.

23. Shaffer F, Ginsberg JP. An Overview of Heart Rate Variability Metrics and Norms. Front Public Health. 2017; 5: 258.

24. Tiselius HS. A biochemical basis for grouping of patients with urolithiasis. Europ Urol. 1978; 4: 241-249.

25. Vis'tak HI, Popovych IL. Vegetotropic effects of bioactive water Naftussya and their endocrine and immune support in female rats [in Ukrainian]. Medical Hydrology and Rehabilitation. 2011; 9(2): 39-57. 